\title{
Extreme High Resolution Imaging of Uncoated Cells in a DualBeam ${ }^{\text {TM }}$
}

\author{
Jessica L. Riesterer ${ }^{1}$, Claudia S. López, ${ }^{2}$ and Eric Barklis ${ }^{2}$ \\ 1. FEI Company, Hillsboro, OR USA \\ 2. Oregon Health and Science University, Department of Molecular Microbiology and Immunology, \\ Portland, OR USA
}

Conductive coatings are often needed to prevent sample charging during SEM viewing. However, coatings that are too thick can obscure small critical features and immune-gold markers, and add time to sample processing. Using the Elstar ${ }^{T M}$ SEM column of the FEI Helios NanoLab ${ }^{T M}$ 650, mammalian cells were imaged as-grown to glass, silicon, aluminum, and paper substrates without the need for conductive coating. Using conditions specific for each substrate material, uncoated cellular bodies, multivesicular exosomes showing roughly spherical shape with various diameters, and cellular protrusions, such as filopodia were imaged. It is possible that the exosome agglomeration observed in these samples might be due to the drying process before the SEM analysis. Cells were not stained prior to viewing.

Human embryonic kidney HEK 293 T cells grown in complete DMEM media suplemented with 10\% Fetal Serum Bovine were seeded onto various sterile substrates previously treated with poly-L-lysine $0.1 \%(\mathrm{w} / \mathrm{v})$ for 5 minutes at room temperature. These cultures were then grown for 48 hours at $37{ }^{\circ} \mathrm{C}$ in a $5 \% \mathrm{CO}_{2}$ atmosphere and then processed for SEM viewing. Briefly, cells were washed in phosphate buffer pH7.4 followed by fixing with cold 4\% paraformaldehyde (w/v) in phosphate buffer for 20 minutes. These cells were then chemically dehydrated in a graded series of ethanol/ $\mathrm{H}_{2} \mathrm{O}$ up to $100 \%$ ethanol, followed by graded series of hexamethyldisilazane/ethanol. After this last step, the samples were air-dried and adhered onto standard $12 \mathrm{~mm}$ SEM stubs using silver paint.

Several key beam conditions contributed to acquiring successful images, as shown in figure 1. Low accelerating voltage $(350 \mathrm{eV}-2 \mathrm{keV})$ and moderate beam current $(50-200 \mathrm{pA})$ were used. Despite not staining the samples, backscattered electron (BSE) imaging gave the best contrast and clearest details. The OHSU Helios NanoLab ${ }^{T M} 650$ is equipped with two types of detectors capable of BSE imaging: 1) a through-the-lens detector (TLD) and 2) a dedicated backscatter (DBS) detector. Both were used with inverted contrast to collect crisp images.

SmartSCAN ${ }^{T M}$ features coupled with beam deceleration allowed high-quality charge-free imaging of the cells. SmartSCAN ${ }^{\mathrm{TM}}$ includes line integration, scan interlacing, frame averaging, and drift corrected frame integration (DCFI), and is embedded within the FEI microscope user interface. This unique suite of features allows reduction of noise in images, drift mitigation. The appropriate value for each of these options depended on the substrate being imaged. Line integration was never set to more than 8 lines. Scan interlacing and frame averaging ranged from 1-8 frames; DCFI ranged from 2-32 frames. Applying beam deceleration, also known as stage bias, between 50-750 V allowed further charge mitigation.

Comparing cells grown on non-traditional surfaces versus glass slides showed no significant differences on the cell surfaces. However, contrast enhancement in BSE images improved when using silicon and aluminum substrates due to the larger z-contrast difference. Therefore, growing cells on various types of conductive samples also serves as another viable option for charge mitigation and contrast enhancement. The use of a paper substrate gives a more three-dimensional look to the cells, more closely resembling real life. 
References:

[1] V. Sokolova et al, Colloids and Surfaces B: Biointerfaces 87 (2011), p. 146.

[2] R. Derda et al, Proc. Natl. Acad. Sci. USA 106 (2009), p. 18467.

[3] G. Wrobel et al, J. R. Soc. Interface 5 (2008), p. 213.

[3] The authors acknowledge funding from the National Institutes of Health, Grant Numbers GM060170 and GM101983. Brandon Van Leer from FEI Company is thanked for his many useful discussions and contributions to this work.

a)
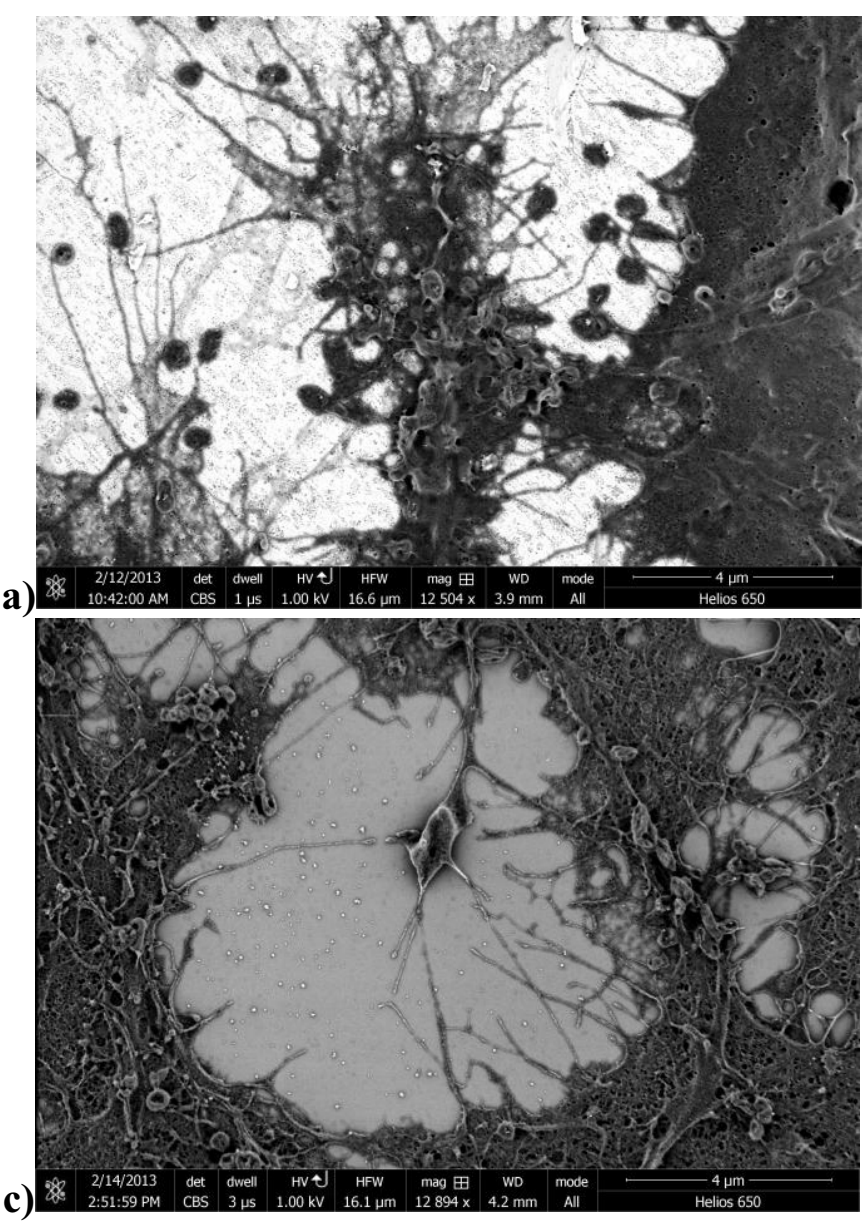
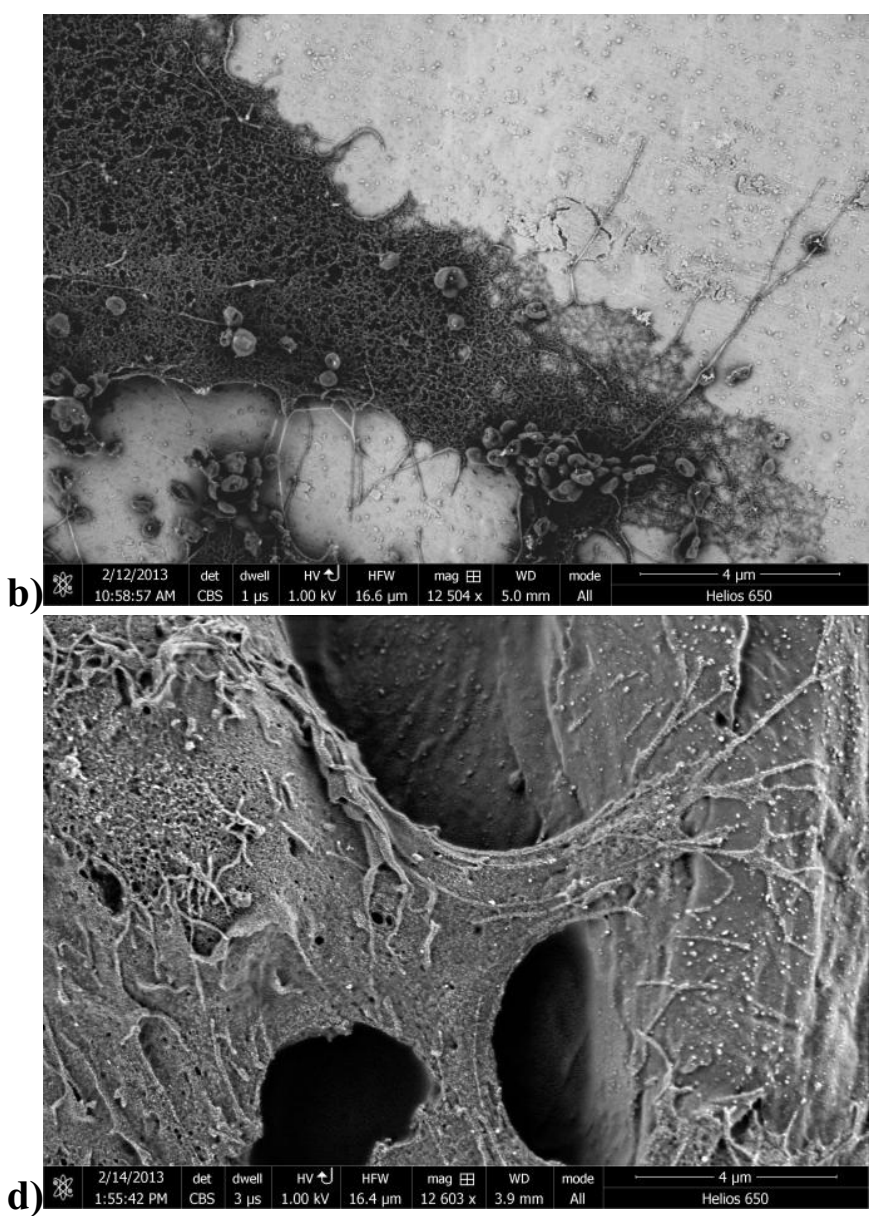

Figure 1. Using SmartSCAN features, a DBS detector, and beam deceleration, high-quality BSE imaging was possible of uncoated cells on a) silicon, b) aluminum, c) glass, and d) cellulose paper substrates. Images were collected using the DBS detector, and scale bars on all images read $4 \mu \mathrm{m}$. Fine details on the filopodia and multivescular exosomes are clearly visible. 